\title{
PJ ZEON Award for outstanding papers in Polymer Journal 2020
}

\author{
Keiji Tanaka, Editor-in-Chief ${ }^{1}$
}

Received: 10 March 2021 / Accepted: 10 March 2021 / Published online: 7 June 2021

(c) The Society of Polymer Science, Japan 2021

The three winners of the 2020 PJ ZEON Award have been announced by the Society of Polymer Science Japan (SPSJ) as follows:

Akihiro Hanafusa (Mitsubishi Chemical Corporation, Japan) for the contribution 'Viscoelastic relaxation attributed to the molecular dynamics of polyrotaxane confined in an epoxy resin network', Vol. 52, No. 10, 2020.

Hiroshi Inaba (Tottori University, Japan) for the contribution 'Cyclic Tau-derived peptides for stabilization of microtubules', Vol. 52, No. 9, 2020.

Toshiki Sawada (Tokyo Institute of Technology, Japan) for the contribution 'Thermally conductive molecular assembly composed of an oligo(ethylene glycol)-modified filamentous virus with improved solubility and resistance to organic solvents', Vol. 52, No. 7, 2020.

Drs. Hanafusa, Inaba, and Sawada received their award certificates and medals. Each winner also received a cash prize of 300,000 JP yen and gave an invited talk based on their respective papers.

On behalf of the editors and editorial board members of Polymer Journal, I wish to congratulate Drs. Hanafusa, Inaba, and Sawada on this honor in recognition of their excellent papers [1-3]. I hope the award will provide encouragement to these young researchers for their bright future careers. Academic profiles of the winners can be found below this announcement.

The PJ ZEON Award started since 2005 as the successor of The PJ Paper Award, which started since 1992.

This PJ ZEON Award is open to all of the first author of papers published in Polymer Journal [4] who is under 38 years of age. We are looking forward to receiving your submissions papers and many applications for the $2021 \mathrm{PJ}$ ZEON Award. Each year the SPSJ selects up to three most outstanding papers published by young authors in Polymer

Keiji Tanaka

hpj@spsj.or.jp

1 Professor, Kyushu University, Fukuoka, Japan
Journal, as recommended by the selection committee and board of directors of the SPSJ. Those who are interested should go to the SPSJ website (https://main.spsj.or.jp//c5/pj/ pj.htm) for further information. Finally, we express our sincere appreciation to Zeon Corporation for their generous sponsorship of this award.

\section{About the winners}

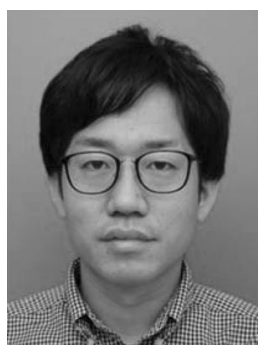

\section{Akihiro Hanafusa}

Akihiro Hanafusa received his MS degree in 2010 from Osaka University. He joined Mitsubishi Rayon Corporation (now Mitsubishi Chemical Corporation) in 2010. At present, he works in collaboration with the University of Tokyo under the supervision of Professor Kohzo Ito. His research interest is the toughing mechanism of polymer materials.

About the award article: The authors reported peculiar viscoelastic relaxation of polyrotaxane (PR) dispersed homogeneously in a cross-linked epoxy resin. This viscoelastic relaxation is attributed to the fluctuation of polyethylene glycol (PEG) in the glassy poly- $\varepsilon$-caprolactone (PCL)-grafted $\alpha$-cyclodextrins (CDs) confined in the epoxy matrix. It was revealed that the mobility of the PEG chain was drastically suppressed by the confinement of the epoxy network. In addition, the reduction of the coverage of CDs in PR decreases the temperature of the relaxation. This result suggests that the molecular dynamics of PR in epoxy resins can be controlled by the coverage of CDs. Furthermore, the viscoelastic relaxation ascribed to PEG enhances the toughness of the epoxy resin under uniaxial stretching. 


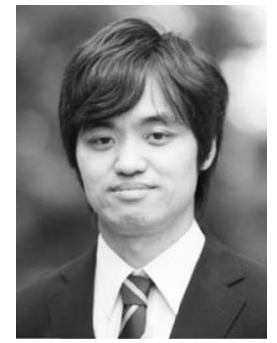

\section{Hiroshi Inaba}

Hiroshi Inaba received Ph.D. degree from Kyoto University in 2015. He was a JSPS research fellow (DC2) in the laboratory of Prof. Susumu Kitagawa (2012-2014). He was a postdoctoral research associate in the group of Prof. Jefferson Chan at University of Illinois Urbana-Champaign, USA (2015-2016). He joined the laboratory of Prof. Kazunori Matsuura at Tottori University as an assistant professor in 2016 and was promoted to an associate professor in 2021. His research interests focused on the design of peptides and proteins for construction of bio-nanomaterials.

About the award article: Microtubules are hollow cytoskeletons and attracted attention as important targets for therapeutics. The authors previously designed a Tauderived peptide (TP) that binds to the interior of microtubules. In this article, the cyclic Tau-derived peptide (TCP) was developed for enhanced binding to tubulin and the stabilization of microtubules. The fluorescently labeled cyclic peptide containing three glycine linkers (TCP3TMR) exhibited a remarkably enhanced binding affinity to tubulin. The cyclic peptide stabilized microtubules by enhancing polymerization and reducing depolymerization.

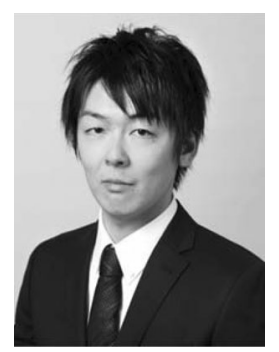

\section{Toshiki Sawada}

Toshiki Sawada received his Ph.D. in bioengineering from Tokyo Institute of Technology in 2010 under the supervision of Professor Hisakazu Mihara. Currently, he works with Professor Takeshi Serizawa and has been involved in The University of Tokyo in 2010 and in Tokyo Institute of Technology from 2012, as an Assistant Professor. He has concurrently served as a PRESTO researcher of the Japan Science and Technology Agency (JST) from 2017. His research interests include soft and/or nanomaterials composed of liquid crystalline viruses.

About the award article: The authors reported chemical modification of M13 phage (a filamentous virus) surfaces with oligo(ethylene glycol) (OEG) to improve solubility and resistance to organic solvents to expand the applicability of M13 phage-based thermally conductive assemblies. High thermal diffusivity of the assemblies composed of M13 phages modified with longer EG chains (EG unit: 10) maintained when the assemblies were prepared using a mixed solvent of water and organic solvents, although assemblies composed of unmodified and shorter EG chain (3 and 6)-modified M13 phages did not. Structural characterization revealed the presence of highly aligned assemblies leading to thermally conductive assemblies in the case of the longer EG chain-modified M13 phages.

Publisher's note Springer Nature remains neutral with regard to jurisdictional claims in published maps and institutional affiliations.

\section{References}

1. Hanafusa A, Ando S, Ozawa S, Ito M, Hasegawa R, Mayumi K, et al. Viscoelastic relaxation attributed to the molecular dynamics of polyrotaxane confined in an epoxy resin network. Polym J. 2020;52:1211-21.

2. Inaba H, Nagata M, Miyake KJ, Kabir AMR, Kakugo A, Sada K, et al. Cyclic Tau-derived peptides for stabilization of microtubules. Polym J. 2020;52:1143-51.

3. Sawada $T$, Tsuruoka $T$, Ueda $N$, Marubayashi $H$, Nojima $S$, Morikawa J, et al. Thermally conductive molecular assembly composed of an oligo(ethylene glycol)-modified filamentous virus with improved solubility and resistance to organic solvents. Polym J. 2020;52:803-11.

4. Tanaka K. PJ ZEON Award for outstanding papers in Polymer Journal 2019. Polym J. 2020;52:551-3. 